\title{
Carcinoma primario do pulmão
}

\author{
Estudo Anatomo-pathologico apresentado \\ á Sociedade Arnaldo Vieira de Carvalho \\ por Mauricio Dereira Lima, do 6. ${ }^{\circ}$ anno \\ da Faculdade de Medicina de S. Paulo.
}

$\mathbf{L}$

ONGE vão os tempos em que um carcinoma do pulmão constituia uma surpresa de autopsia. Clinica e anatomia-pathologica progrediram. Tanto que hoje são raros os medicos que não contêm na sua carreira mais de um diagnostico brilhante dessa affecção. E rarissimos os laboratorios de pathologia que, por sua causa, não tenham tido o desprazer de infirmar um diagnostico de tuberculose pulmonar, ou de tumor liquido do mediastino.

Maior frequencia não quer dizer, porem, esgotamento do assumpto. Ao contrario. Cada novo caso levanta novas difficuldades, cujo estudo e resolução constituem outros tantos andares do arranha-céu que é o problema do cancer.

Não pretendemos, com o ligeiro estudo que se segue, erguer paredes, collocar vigas solidas sobre que outros possam apoiar ulteriormente suas construcções. O edificio é elevado e perigoso. Muita gente tem cahido dahi, e, se na queda não parte o pescoço, soffre ao menos contusões desagradaveis. Vamos ficar no pavimento terreo, mais ou menos estavel, e contemplar o resto por um oculo. Processo burguez, de que se utiliza a humanidade ao contemplar a solução de todos os grandes problemas.

Antes do mais, ajuize-se da frequencia do carcinoma do pulmão pelos seguintes dados:

Willi Marchesani, revendo o archivo do Instituto de Pathologia de Innsbruck, encontrou 26 casos de ca. do pulmão entre 13.367 necropsias praticadas naquelle instituto de 1887 a 1922 ( 35 annos).

Hansemann, em 7.790 necropsias, observou 511 carcinomas de varios orgãos; dos quaes 16 primitivos do pulmão.

Entre as 16.047 necropsias praticadas por Lavinovitch em Leningrado, ha 61 casos de ca. do pulmão $(0,38 \%)$.

Menétrier observou 6 casos em 2.500 necropsias.

Cotton, Cramer e Saloz (Hôpital Cantonnal de Genève) assignalaram 29 carcinomas do pulmão num periodo de 20 annos.

Dos 16.578 casos de carcinoma encontrados por Karrenstein e Aler, 168 eram do pulmão; uma percentagem de $1 \%$. 
Kaufmann dá uma percentagem de 1,83\%

Entre nós. No Instituto Anatomo-Pathologico da nossa Faculdade praticaram-se de 1916 a 1925, 900 necropsias, entre as quaes ha 92 casos de tumores malignos, sendo 65 carcinomas. Carcinomas primitivos do pulmão 2 (A 45-1916 e A 884-1925). A percentagem é de $3 \%$ dos 65 carcinomas, e $0,22 \%$ das 900 necropsias. Em compensação ha 24 carcinomas do estomago, ou $37 \%$

Ainda quanto á frequencia dos carcinomas primitivos do pulmão, ha um facto interessante a assignalar. Essa frequencia tem augmentado ultimamente. Assim, os 26 casos encontrados por Marchesani estão distribuidos da seguinte maneira:

$$
\begin{aligned}
& \text { De 1887-1896 } 4 \text { casos, em } 1.940 \text { necropsias, ou } 0,26 \% \\
& \text { "1896-1906 } 6 \text {, , } 3.337 \quad, \quad, 0,18 \% \\
& \text { ", 1906-1916 6 ", , } 4.754 \quad, \quad, \quad 0,13 \% \\
& \text { ", 1916-1922 10 " " " } 3.336 \text { " " , " } 0,3 \% \\
& \text { (de } 1 / \mathrm{I} / 22 \text { a } 1 / \mathrm{X} / 22 \text { houve } 3 \text { casos em } 210 \text { necropsias, }
\end{aligned}
$$

No Bellevue Hospital (N. Y City) foram registrados de 19071919 (12 annos), 5 casos de ca, do pulmão. Nos 2 annos seguintes registraram-se 7 novos casos.

De 5 casos encontrados no Peter Bent Brigham Hospital, 2 o foram em 1921; 2 em 1923; 1 em 1924. De 1913-21 não foi assignalado caso algum.

Muitos autores attribuiram esse augmento na frequencia dos carcinomas pulmonarres ás epidemias de grippe que se tem repetido ultimamente. Outros, como Fried, dizem que o facto é explicavel pelo maior cuidado com que se praticam as autopsias hoje em dia, sendo assim o controle do diagnostico clinico muito mais rigoroso. Dos 10 casos que esse autor observou recentemente, 2 traziam o diagnostico de tuberculose pulmonar chronica; 1 de psychose alcoolica; 1 de lymphosarcoma; 2, indeterminados; 2 de ca. do pulmão.

$\mathrm{O}$ erro é eterno no homem. Bom que se lembrem disso os que censuram os modernos clinicos por se embaraçarem diante de certos casos "complicados" apezar de disporem de um arsenal semiotico infinitamente mais perfeito e abundante que os antigos mestres. A memoria destes é sagrada. Seria pretensão tola querer tirar-lhe qualquer valor. Mas, quantos erros um estudo anatomo-pathologico acurado não viria desvendar nos diagnosticos de outrora. E quantos ainda não desvendará para o futuro! Alem disso, a propria complicação semilogica permittiu um estudo mais aprofundado das molestias, e consequente difficuldade nos diagnosticos exactos. O clinico avança mais cauteloso, consciente do perigo. Evita muito mais os passos falsos.

Curioso, se fossemos censurar os generaes modernos por empregarem o 150 ou o 420, em vez de engendrarem estratagemas semelhantes ao de Ulysses para penetrar em Troia. 
Vamos passar á exposição do caso que estudamos no Instituto de Anatomia-pathologica da nossa Faculdade, mercê da gentileza e esclarecida orientação do illustrado professor Cunha Motta.

Resumo de historia clinica:-

J. R. hespanhol, 46 annos, sexo mascullino.

Um mez antes de entrar para o hospital começou a sentir fadiga após qualquer esforço. Tinha uma tosse secca que muito o incommodava. Notou que o rosto tornava-se edemaciado. Nos ultimos dias de vida apresentou dysphonia, aphonia, dysphagia, que se foram accentuando gradualmente. Dores fortissimas tornavam necessario o emprego diario da morphina.

Exame physico - Pupilla esquerda mais dilatada que a direita, e reagindo á luz lentamente. Apagamento das fossas supracliviculares. Abundante circulação collateral venosa nos territorios da cava superior, inferior e da azygos, mais accentuada á direita. Ganglios inguinaes palpaveis. Hernia inguinal de ambos os lados Hemithorax esquerdo, diminuição das incursões respiratorias na base, massicez, signal de Ramond. Abolição quasi completa do fremito nessa mesma região, assim como diminuição accentuada da respiração. Na parte superior do hemithorax, sopro expiratorio de tonalidade aguda. Pulso radial - 100 por minuto. Diagnostico clinico - Aneurysma da aorta (porção ascendente).

Protocollo da autopsia (redação de E. F. Martins Passos).

Data da autopsia - 17/IV/25. Autopsiantes — Prof. Lambert e Dr. M. Sainati.

\section{DIAGNOSTICO ANATOMO-PATHOLOGICO}

Carcinoma primario do pulmão direito; carcinoma metastatico dos ganglios mediastinaes; pleurite fibrino-purulenta; arterio esclerose accentuada da aorta; esteatose e hemangioma multiplo do figado; kysto sebaceo da pelle; hydrocele; thromboses multiplas das arterias pulmonares; focos hematopoieticos de baço; hyperplasia fibrosa circumscripta da prostata.

Passamos a transcrever a parte do protocollo em que se dá conta do estado da cavidade thoracica e dos orgãos que ahi se achavam:

Cavidade pleural direita contem grande quantidade de liquido (2 litros e meio), fibrino purulento. As pleuras se acham espessadas e revestidas de uma camada de fibrina com grande numero de adherencias. O pulmão se acha recalcado para o mediastino de modo que 


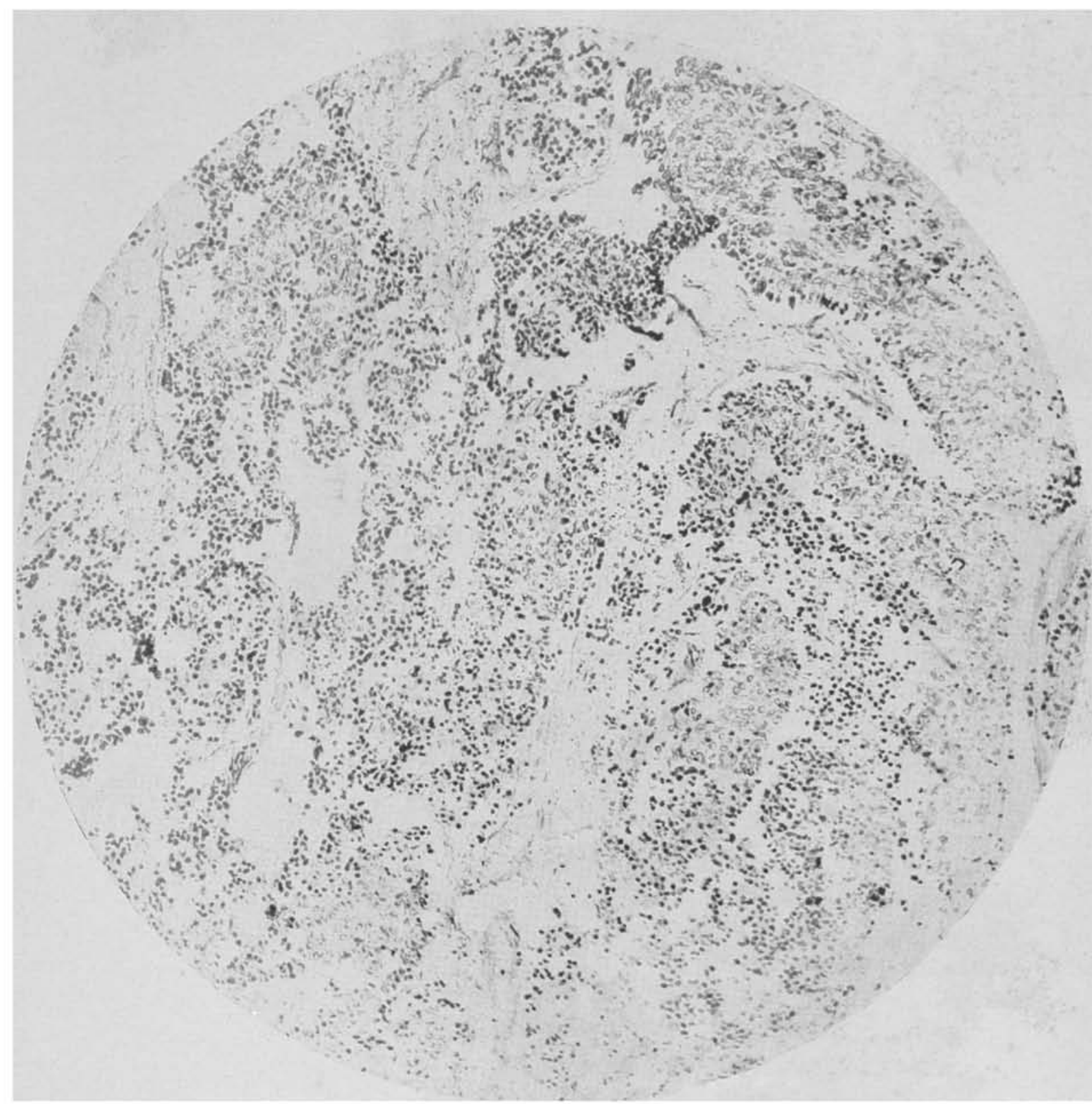

CORTE DO TUMOR MOSTRANDO AS CELLULAS DE NUCLEOS PEQUENOS, CHROMATINA DENSA

E FINA ORLA PROTOPLASMATICA. 

entre o pulmão e a pleura costal existe um grande espaço no qual se accumula grande quantidade de liquido.

Cavidade pleural esquerda. Essa cavidade se acha livre de exsudato, as pleuras lisas e brilhantes e sem adherencias.

Pericardio - liso brilhante e madreperlaceo. Pela sua abertura verifica-se que em sua cavidade existe 40 a $50 \mathrm{cc}$. de um liquido de cor amarello avermelhada. Pela abertura da arteria pulmonar não se encontram embolos. No mediastino anterior, fazendo corpo com o pulmão direito verifica-se a presença duma grande massa de consistencia dura, constituida por grande quantidade de ganglios infartados, agglomorados. Abrindo-se essa massa, verifica-se que ella não tem relação com as paredes da trachea, esophago e aorta, pois, estes orgãos mostram a sua luz perfeitamente normal.

Pulmão esquerdo - Peso 420 grs. Volume normal, forma pyramidal, coloração cinzento azulada e rosea no lobulo superior. Consistencia normal. Os ganglios do hilo mostram-se infartados. Bronchios de calibre normal. Mucosa de coloração acinzentada, não espessada e sem secreção. Superficie de corte - coloração vermelho escura; pela pressão obtem-se um liquido de cor vermelho, espumoso.

Pulmão direito - Peso 550 grs. Forma quadrangular. A pleura visceral é opaca, de cor branco amarellada, apresentando superficie irregular devido ao exsudato fibrino purulento sobre ella depositado.

Consistencia augmentada irregularmente, mostrando pela palpação zonas duras e resistentes que alternam com outras de consistencia mais ou menos normal. Ao corte vemos o tecido de cor branco amarellada e cinzento avermelhada. Abertos os bronchios, bem como a trachea, vê-se que elles mostram a sua luz normalmente revestida por mucosa intacta e levemente hyperemica.

Pelo exame dos cortes histologicos do tumor, observamos ser elle constituido na sua maior parte por cellulas do nucleo pequenos, com chromatina densa, cytoplasma reduzido a fina orla peripherica, de coloração basophila. Essas cellulas se dispõem entre feixes collagenos, de espessura variavel, que se entrecruzam em todas as direcções. Mas, alem dellas, ha focos de agglomerados cellulares constituidos por elementos do typo francamente epithelial, polymorphos, de nucleos vesiculoso, nucleolo visivel e cytoplasma apreciavel. Essas cellulas são planas e não deixam ver entre si nem estroma conjunctivo, nem vasos. Não ha tendencia a formações perlaceas. As cellulas do typo epithelial acham-se reproduzidas em todas as metastáses ganglionares do tumor.

O aspecto macrescopico do tumor e a profusão das suas metastases mediastinaes, levaram, antes do exame dos preparados histologicos, as classificarem-n'o como um lymphosarcoma do mediastino. Aliás, como acima vimos, é em grande parte constituido por elementos que apresentam todos os caracteres dos lymphocytos (nucleo pequeno, chromatina densa, occupando quasi toda a cellual, sendo o 
protoplasma reduzido a fina orla peripherica). Mas a existencia de cellulas francamente epitheliaes, de nucleo vesiculoso, repetindo-se em todas as metastases, veio tirar todas as duvidas quanto á classificação do tumor. Pois é regra quanto mais complexo um neoplasma, tanto mais simples as suas metastases e recurrencias. Ahi melhor podem ser estudados os seus carecteres primitivos. Trata-se portanto, de um carcinoma polymorpho. Caracter não raro dos tumores do apparelho respiratorio e que demonstra uma grande anaplasia nas cellulas tumoraes. Segundo o prof. Cunha Motta, a anaplasia dessas cellulas de nucleo pequeno é devida á existencia de estroma conjunctivo proliferado em que ellas se encontram, que, de certo modo as comprimindo, impede seu completo desenvolvimento e expansão.

\section{H IS TOGENESE}

Ewing e a maioria dos autores admittem 3 typos de carcinomas pulmonares. a) Carcinomas derivados de epithelio bronchial; b) carcinomas derivados de epithelio das glandulas mucosas bronchiaes; c) carcinomas derivados de epithelio alveolar. 'E' evidente quanto uma tal classificação é imprecisa. Admitte-se que um tumor seja de origem bronchial, ou alveolar, porque occupa na sua maior parte um bronchio ou o parenchyma pulmonar. Ou ainda, porque as suas celluas têm a mesma morphologia que. as do epithelio bronchial ou alveolar. Mas, já o dissemos, o polymorphismo é caracter commum dos epitheliomas pulmonares. E em geral, quando se procede á autopsia, o tumor acha-se bastante diffundido para que a sua posição primitiva seja determinada. Para chegar-se a resultados seguros, e essa é a opinião de Marchesani, necessario seria estudar methodicamente todos os pulmões em que se pudesse suspeitar um processo carcinomatoso, para assim surprehender o neoplasma nas suas primeiras phases. Ora, taes estudos seriam dispendiosos, fastidiosos Impraticaveis para espiritos latinos.

Apezar de tudo, ainda é a classificação de Ewing a mais simples e a melhor. Nos carcinomas do primeiro typo a proliferação tumoral se dá principalmente na mucosa dos bronchios, dahi se estende ás paredes bronchiaes, septos e pleura. Começa o processo em geral na bifurcação de um bronchio e propaga-se para cima, á trachea, para baixo, aos bronchios menores. Ha geralmente projecções papillares para o interior dos bronchios; suas paredes são espessadas e o parenchyma visinho é invalido. Todavia não ha, em geral, produção de um tumor diffuso. Kaufmann chama a attenção para os tumores estenosantes ou ulcerantes, que, situados nos bronchios principaes, podem passar despercebidos durante a autopsia. Quanto á estructura histologica, os tumores bronchiaes se dividem em escamosos e cylindro-cellulares. Os primeiros são mais ou menos uniformes, ao passo 


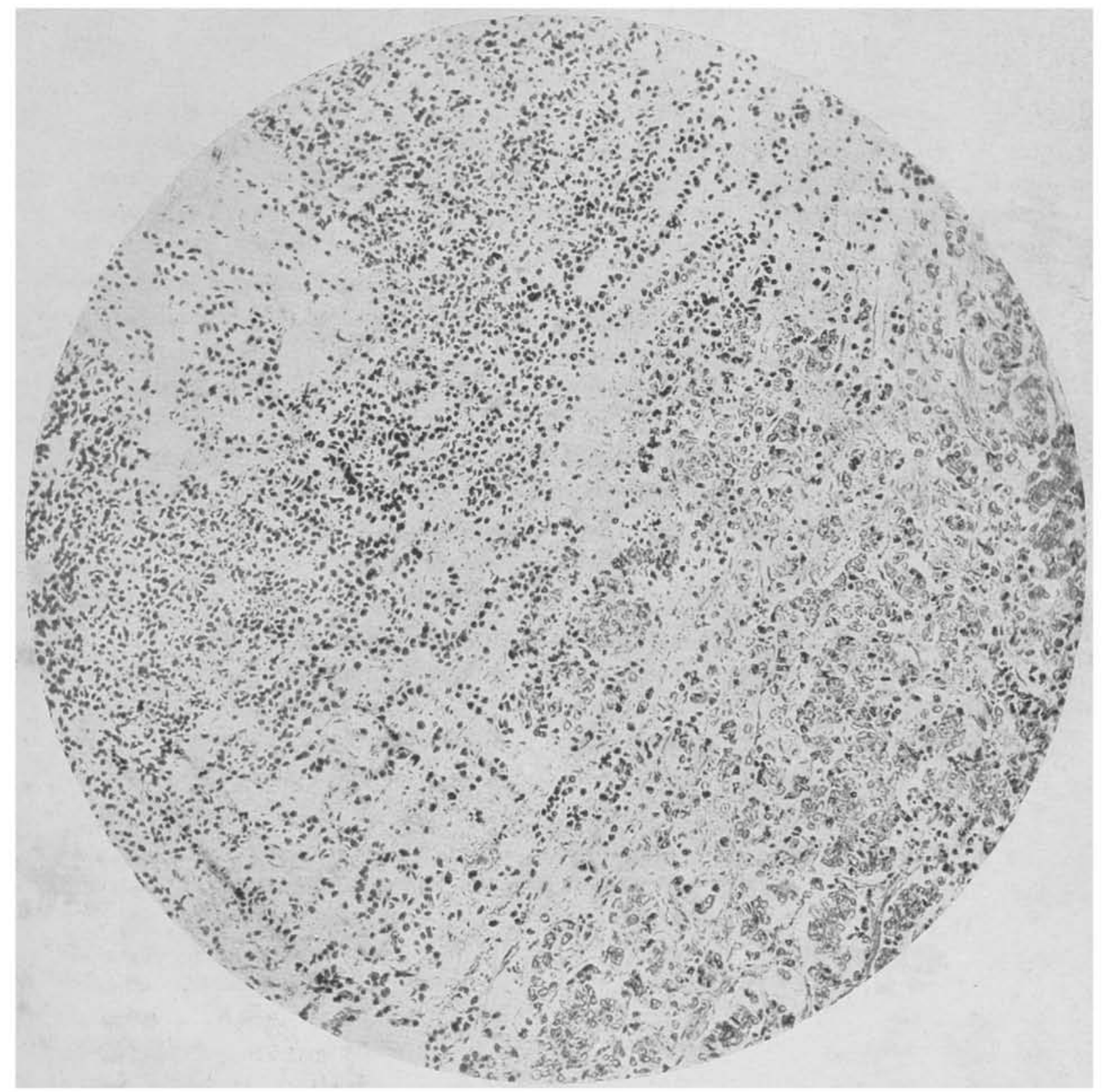

CORTE DO TUMOR, APRESENTANDO CELLULAS

DE ASPECTO LYMPHATICO E CELLULAS

FRANCAMENTE EPITHELIAES. 

que os cylindricos variam, podendo apresentar uma estructura adenocarcinomatosa, papillar, etc.

Nos carcinomas derivados do epithelio das glandulas bronchiaes a proliferação se inicia especiamente na submucosa do bronchio. A mucosa permanece intacta. Ha estenoze bronchial em logar de bronchoectasia. Seguida em geral de uma atelectasia do tecido pulmonar servido pelo bronchio lesado. O parenchyma pode ser infiltrado. Estructura-adenocarcinomatosa, ou alveolar, lembrando as glandulas mucosas.

Os carcinomas derivados do epithelio alveolar podem ser diffusos ou nodulares. Os diffusos chegam a occupar um lobo inteiro. Ahi o parenchuma pulmonar adquire uma consistencia solida; ha formação de cavernas pela necrose das partes centraes. $\mathrm{O}$ processo assemelha-se á pneumonia na phase de hepatização cinzenta. A pleura é geralmente envolvida. Hstologicamente são compostos por cellulas cubicas, cylindricas ou chatas. Os nodulares, assemelhando-se muito á tuberculose, podem estender-se pelos dois pulmões. Apresentam no seu centro zonas de necrose. São formados por alveolos dilatados, cheios de projecções palpillares.

O tumor que descrevemos nas paginas supra parece ser derivado do epithelio alveolar. Porque- pelo protocollo da autopsia e pelo relatorio histopathologico ficou especificado que a massa tumoral deixa a luz dos bronchios completamente livre; $e$ as camadas mucosa, muscular e fibrosa não possuem cellulas tumoraes. Isto é sufficiente para excluil-o da classe dos derivados do epithelio bronchial, ou do epithelio das glandulas mucosas. Em favor da hypothese de que derive do epithelio alveolar, falam ainda o seu aspecto diffuso e o seu polymorphismo cellular.

Já nos referimos á explicação dada pelo Prof. Cunha Motta á anaplasia, ao polymorphismo do tumor que estudamos. E' simples, clara, e dispensaria ulteriores commentarios. Mas, não poderiamos passar em silencio os estudos feitos sobre os tumores heterotypicos das vias respiratorias, principalmente carcinomas espino-cellulares com corneificação. A' sua histogense e etiologia estão muito ligados os processos de metaplasia do epithelio. Metaplassia neste caso é a substituição do epithelio vibratil bronchial ou alveolar por um epithelio pavimentoso polyestratificado. Este phenomeno tem sido observado em condições differentes por varios autores, ou seja em casos apparentemente normaes, ou em seguida a processos pathologicos chronicos, taes como a tuberculose, a syphilis, as bronchoectasias. Askanazy encontrou "modificações de catharros metaplasticos" 38 vezes em 90 casos mortaes de grippe durante a grande epidemia de 1918. Notou que o epithelio da superficie em larga extensão ou em zonas limitadas se transformava em epithelio pavimentoso polyestratificado, havendo mesmo em um caso formação de papillas, sem, todavia, a corneificação das camadas superficiaes. 
Siegmund descreveu em varios casos uma proliferação das cellulas basaes do epithelio em descamação, constituindo-se então uma camada de cellulas fusiformes, longas ou curtas, com protoplasma escasso, nucleo oval ou redondo, que chamou epithelio de transição (übergangsepithel). Admitte, com Goldzieher, que este epithelio de transição possa evoluir em um epithelioma malpighiano typico.

Teutschlander demonstrou esse facto estudando a broncho-pneumonia experimental dos ratos. Havia nesses casos corneificação do epithelio.

Para Siegmund todo o cancer heterotypico das vias aereas deriva dum epithelio de transição. Não considera necessaria uma completa metaplasia, nem mesmo para a explicação dos ca. de cellulas chatas, que na sua opinião aproximam-se muito mais dos carcinomas basocellulares que dos espinocellulares. E conclue- "não nego tão pouco a possibilidade da origem de taes tumores de epithelio completamente metaplastico, como tambem concordo com Askanazy em que ca-espinocellulares puros possam originar-se de cellulas basaes, não ulteriormente modificadas" Os carcinomas alveolares derivariam tambem, para Siegmund, do epithelio de transição, pois em varios casos de grippe encontrou os alveolos forrados por esse epithelio, resultante da proliferação do epithelio bronchial.

Teutschlander admitte que o epithelio ciliado da trachea, que tem a mesma origem embryonaria que o ephithelio polyestratificado do esophago, permaneça num gráo de evolução inferior ao deste ultimo. Em condições pathologicas, como nas inflammações chronicas, o epithelio em se regenerando, continua a sua differenciação (prosolasia), podendo esta parar em qualquer dos estados- de cellulas basaes, epithelio chato ou formas de transição entre ambos (tumores. polymorphos). De modo que a "metaplasia segue um caminho predeterminado pela historia do desenvolvimento". Desapparecendo as exitações determinantes da metaplasia, a regeneração physiologica retoma o seu curso. Continuem, porém, essas excitações a energia de multiplicação de epithelio em regeneração pode dar logar á formação de tumores epitheliaes benignos ou malignos. Os ca. de cellulas basaes, polymorphas, planas com corneificação, originam-se, portanto, de um epithelio regenerado pathologicamente.

De accordo com essas ideas tudo que é capaz de produzir metaplasia, o é, em determinadas condições, de provocar a formação de neoplasmas. E eis porque, constatado o facto, embrenharam-se os autores pela floresta das hypotheses, a descobrir estimulos metaplasticos. Encontraram a poeira, as bronchoectasias, a tuberculose, a grippe, a maior accessibilidade do bronchio direito, a syphilis, etc. Nenhum resolveu a questão. Por isso convem quedarmo-nos. O problema da etiologia dos carcinomas começa agora a amadurecer. Não o toquemos, para não deixar nodoas na sua superficie. 


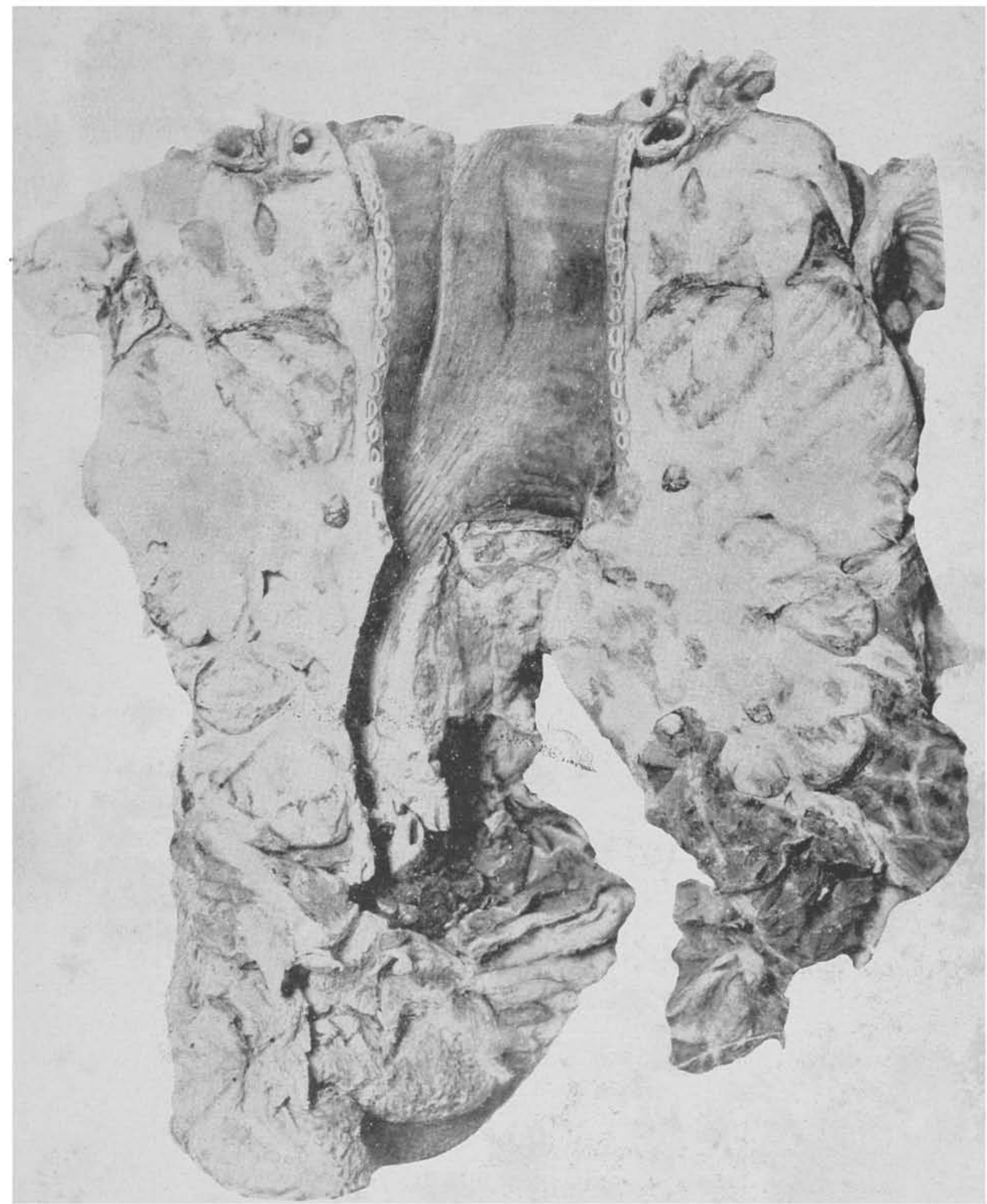

ASPECTO MASCROSCOPICO DO TUMOR E DAS METASTASES MEDIASTINAES. 



\section{B I B L IO G R A P I A}

BöCKER - Zur Kenntniss der primären Lungenkarzinome - Virch. Arch. Vol. 202-1910.

Cincinato Leme Ferreira - Contribuition à l'étude du Cylindrome et $d u$ Carcinome primaire de la Tracbée - Thèse de Genebra, 1919.

ERnst - Virchones Pathologie einst und jetzt - Virch. Arch. Vol. 235-1921.

EwING - Neoplastic Diseases.

FRIED - Primary Ca. of the lungs -- Arch. of Int. Med. Vol 35-1925.

R. LAMBERT - Four Cáses of primary Carcinoma of the Lung Proc. of the N. Rork Pathological Soc. Vol. XV-1915.

Malassez - Tumeur du poumon - Arch. de Physiologie - 1876.

MARCHESANI - Ueber den primären Bronchialkrebs - Frankf. Zeitsch. für Pathologie - Vol. 30-1924.

MEYER - Ein Fall von Epithelmetaplasie und metaplasierenden Karinom des rechten Hauptbronchus nach Grippe - Frankf. Zeitsch. für Path. Vol. 27-1922.

Muller - Two cases of primary ca. of the lung - Proc. of the N. York Pathological Soc. Vol. XVII n. ${ }^{0}$ 1-5.

Siegmund - Krebsentwicklung in Broncbiektsen - Virch. Arch. Vol. 236-1922.

Stillmann - Ca. of the Bronchus - Proc. of the N. York Path. Soc. Vol. XX-1920.

\section{PHARMACIA LANGE}

PREÇOS ESPECIAES PARA MEDICOS E ESTUDANTES DE MEDICINA

Pedidos por Telephone - Central 2223 RUA VERGUEIRO, 10 S. PAULO 